ISSN 1991- 8690

website: http:// jsci.utq.edu.iq
الترقيم الدولي 8690 - 1991

Email:utjsci@utq.edu.iq

\title{
Semi Regular and semi weak Regular spaces in intuitionistic topological spaces
}

\author{
Luma S. Hany
}

Department of Mathematics - college of education for women - University of Tikrit

\section{$\underline{\text { Abstract }}$}

The aim of this paper, giving a new definition of semi-Regular and semi weak Regular spaces in Intuitionistic topological space in additional to studying some correlated equation with several example.

Keywords: Intuitionistic set, intuitionistic fuzzy topological spaces, Regular, Semi-Regular, semi weak regular.

$$
\begin{aligned}
& \text { الفضاءات شبه المنتظمة وشبه المنتظمة الضعيفة في الفضاءات التبولوجية الحدية } \\
& \text { لمى سعد عبد الباقي } \\
& \text { قسم الرياضيات - كلية التربية للبنات - جامعة تكريت }
\end{aligned}
$$

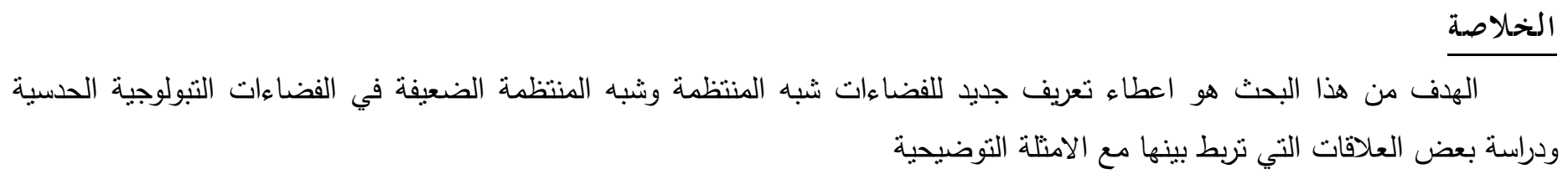

\section{1- Introduction:}

The concepts of fuzzy set was introduced by Zadeh (1965) in his classical paper. A fuzzy set A in X is afunction from $X$ to the unit interval $[0,1]$. For any $x$ in $X$ the number $A(x)$ is called the membership degree of $\mathrm{x}$ in $\mathrm{A}$.

After the discovery of the fuzzy subset, much attention has been paid to generalize the basic concept of classical topology in fuzzy setting and thus a modern theory of fuzzy topology has been developed .The notion of fuzzy subset naturally plays avery significant role in the study of fuzzy topology which was introduced by Chang C.L. in (1968) also Bayhan and Coker in (1996).

In 1983, Atanassov introduced the concest of "Intuitionistic fuzzy set"and Atanassov 1984 and 1986 using a type of generalized fuzzy set Coker 1997 , while a fuzzy set gives a degree of membership of an elementin a given set intuitionistic fuzzy sets given both degree of membership and a non-membership .Both degrees belong to the interval $[0,1]$, and their sum should not exceed 1 , formally an intuitionistic fuzzy set $\mathrm{A}$ in X was defined as an object of the form $A=\left\{\left\langle x, \mu_{A}(x), \gamma_{A}(x): x \in X\right\rangle\right\}$ where $\mu_{\mathrm{A}}(\mathrm{x})$ is called the degree of membership of $\mathrm{x}$ in $\mathrm{A}$ and $\gamma_{\mathrm{A}}(\mathrm{x})$ the degree of non- membership of $\mathrm{x}$ in $\mathrm{A}$, And $0 \leq \mu_{A}(x)+\gamma_{A}(x) \leq 1$, for each $x \in X$. [2]. 
After introducing the concept of fuzz sets by Zadeh 1965, several researches were conducted on generalizations of notion of fuzzy set, and after the idea of intuitionistic fuzzy set which the first published by Krassimir Atanassov 1988, also Atanassov with Stoeva 1983, many works appeared in the literature. Later, the concept is used to define intuitionistic fuzzy special sets by [2], and intuitionistic fuzzy topological spaces are introduced by Coker 2000, and Malghan, R.S. with Benchalli,S.S. 1981 also Fora A.A1989 in "Separation axioms for fuzzy spaces "and Separation axioms in intuitionistic fuzzy topological spaces by Bayhan.s. and coker.D. 19996, 2003.

In this paper we introduced and studied the concept of regular and semi-regular space in intuitionistic topological spaces and defend $\mathrm{R}(\mathrm{k})$ where $k \in\{i, i i, i i i, i v\}$, and study the relations of them with proved and given counter examples.Also we introduced and studies the concept of weak regular and weak semi regular space in intuitionistic topological spaces and study the relation of them with proofs and giving counter examples.

\section{2-Preliminaries :}

\section{Definition 2.[1]}

Let $\mathrm{X}$ be a nonempty set .An intuitionistic fuzzy special set $\mathrm{A}$ is an object having the form $\mathrm{A}=<\mathrm{x}, \mathrm{A}_{1}$, $A_{2}>$, where $A 1$ and $A 2$ are subsets of $X$ satisfying $A_{1} \cap A_{2}=\phi$. The set $A_{1}$ is called the set of members of $A$, while $\mathrm{A}_{2}$ is called the set of non-members of $\mathrm{A}$.

\section{Properties 2.2[1]:}

Let $\mathrm{X}$ be a nonempty set and the intuitionistic fuzzy special set $\mathrm{A}$ and $\mathrm{B}$ be in the form $A=<x$, $\left.A_{1}, A_{2}\right\rangle, B=\left\langle x, B_{1}, B_{2}\right\rangle$ furthermore, let $\{A i: i \in \mathrm{I}\}$ be an arbitrary family of intuitionistic fuzzy special sets in $\mathrm{X}$ where $\mathrm{Ai}=<\mathrm{x}, \operatorname{Ai}(1), \operatorname{Ai}(2)>$

$$
\begin{aligned}
& \text { 1- } A \subseteq B \Leftrightarrow A_{1} \subseteq B_{1} \& B_{2} \subseteq A_{2}, \\
& \text { 2- } A=B \Leftrightarrow A \subseteq B \& B \subseteq A,
\end{aligned}
$$

3-The complement of an intuitionistic set $\mathrm{A}$ in $\mathrm{X}$ is denoted by $\bar{A}$ and defined by $A=<x, A_{1}, A_{2}>$ 4- $F A=\left\langle x, A_{1}, A_{1}^{c}\right\rangle,-S A=\left\langle x, A_{2}, A_{2}^{c}\right\rangle$.

5- $\cup A_{i}=\left\langle x, \cup A_{i}^{(1)}, \cap A_{i}^{(2)}\right\rangle, \cap A_{i}=\left\langle x, \cap A_{i}^{(1)}, \cup A_{i}^{(2)}\right\rangle$.

6- $\widetilde{\emptyset}=\langle x, \emptyset, X\rangle, \tilde{X}=\langle x, X, \emptyset\rangle$

\section{Definition 2.3 [1]:}

Let $X$ be a non-empty set, $p \in X$ be a fixed element in $X$, and let $A=<x, A_{1}, A_{2}>$ be intuitionistic set (IS, for short) , intuitionistic point $\tilde{\mathrm{p}}$ in X (IP, for short ) is an intuitionistic set defined by $\tilde{p}=$ $\left\langle x,\{p\},\{p\}^{c}\right\rangle$. The vanishing intuitionistic point $\tilde{\tilde{\mathrm{p}}}$ in X (VIP, for short) in an intuitionistic set defined by $\tilde{\mathrm{p}}=\left\langle\mathrm{x}, \emptyset,\{\mathrm{p}\}^{\mathrm{c}}\right\rangle$.

The IS $\tilde{\tilde{\mathrm{p}}}$ is said to be contained in A $\tilde{\mathrm{p}} \in A$, for short) if and only if $p \in A_{1}$ and similarly $\tilde{\mathrm{p}}$ contained in $\mathrm{A}(\tilde{\mathrm{p}} \in A$, for short $)$ if and only if $\mathrm{p} \notin \mathrm{A}_{2}$. For a given IS $\mathrm{A}$ in $\mathrm{X}$, we may write $A=(\cup\{\tilde{p}: \tilde{p} \in A\}) \cup$ $(\cup\{\tilde{\tilde{p}}: \tilde{\tilde{p}} \in A\})$, and whenever $\mathrm{A}$ is not proper IS ( i.e.if $\mathrm{A}$ is not of the form $A=<x, A_{1}, A_{2}>$ where $\left.A_{1} \cup A_{2} \neq \varnothing\right)$, then $A=\cup\{\tilde{p}: \tilde{p} \in A$ \}hold .

In general, any IS $A$ in $X$ can be written in the form $A=\widetilde{A} \cup \widetilde{\widetilde{A}}$ where $\widetilde{A}=U\{\tilde{p}: \tilde{p} \in A\}$ and $\widetilde{\widetilde{A}}=U$ $\{\tilde{\tilde{p}}: \tilde{\tilde{p}} \in A\}$.

\section{Proposition 2.4[2]:}

Let $\mathrm{X}$ and $\mathrm{Y}$ be two nonempty sets and Let $\mathrm{f}$ be a function from a set $\mathrm{X}$ to a set $\mathrm{Y}$ : 
1- If $B=\left\langle x, B_{1}, B_{2}\right\rangle$ is an IS in Y,then the preimage of $\mathrm{B}$ under $\mathrm{f}$ is denoted by $f^{-1}(B)$ is an IS in $\mathrm{X}$ and defined by $f^{-1}(B)=\left\langle x, f^{-1}\left(B_{1}\right), f^{-1}\left(B_{2}\right)\right\rangle$.

2- If $A=\left\langle x, A_{1}, A_{2}\right\rangle$ is an IS in $X$, then the image of under $f$ denoted $(A)\left\langle y, f\left(A_{1}\right), \underline{f}\left(A_{2}\right)\right\rangle$ where $\underline{f}\left(A_{2}\right)=$ $\left(\mathrm{f}\left(\mathrm{A}_{2}^{\mathrm{c}}\right)\right)^{\mathrm{c}}$.

\section{Definition 2.5[3]:}

An intuitionistic topology on a nonempty set $\mathrm{X}$ is a family $\mathrm{T}$ of an intuitionistic sets in $\mathrm{X}$ satisfying the following conditions:

1- $\widetilde{\emptyset}, \tilde{X} \in T$

2- $\mathrm{T}$ is closed under finite intersections.

3- $\mathrm{T}$ is closed under arbitrary unions.

The pair (X,T) is called an intuitionistic topology space ( ITS, for short). Any element in T is usually called intuitionistic open set (IOS , for short). The complement of IOS in a ITS(X,T) is called intuitionistic closed set ( ICS, for short).

\section{Definition 2.6 [1]:}

Let $(\mathrm{X}, \mathrm{T})$ be an ITS, then :

$$
-T_{0.1}=\left\{F G: G=\left\langle x, G_{1}, G_{2}\right\rangle \in T\right\}
$$

$-T_{0.2}=\left\{S G: G=\left\langle x, G_{1}, G_{2}\right\rangle \in T\right\}, \quad$ are intuitionistic topologies on X .

$-T^{*}=\left\{G_{1}: G=\left\langle x, G_{1}, G_{2}\right\rangle \in T\right\}$.

$$
-T^{* *}=\left\{G_{2}^{c}: G=\left\langle x, G_{1}, G_{2}\right\rangle \in T\right\}, \quad \text { are topologies on X. }
$$

Next, we give the definition of interior and closure of IS A in ITS(X,T).

\section{Definition 2.7[2]:}

Let (X,T) be an ITS and let $A=\left\langle x, A_{1}, A_{2}\right\rangle$ be an intuitionistic subset (ISs , for short) in a set X . the interior (IntA , for short) and closure (ClA , for short) of a set $\mathrm{A}$ of $\mathrm{X}$ are defined:

-IntA $=\cup\{G: G \subseteq A, G \in T\}$.

$-\mathrm{ClA}=\cap\{F: A \subseteq F, \bar{F} \in T\}$.

In other words, The IntA is the largest intuitionistic open set contained in $\mathrm{A}$, and $\mathrm{ClA}$ is the smallest intuitionistic closed set contained $\mathrm{A}$, i.e. Int $A \subseteq A$ and $A \subseteq C l A$.

Now, we give this remark and explain the converse is not true in general .

\section{Remark 2.8:}

Let $(\mathrm{X}, \mathrm{T})$ be an ITS, and $\mathrm{U}, \mathrm{V} \in T$ then ,if $U \cap V=\widetilde{\emptyset}$ then, $U \subseteq \bar{V}$ or $V \subseteq \bar{U}$.

We have example to explain converse case is not true.

\section{Example 2.9:}

Let $=\{a, b\}, A=\langle x,\{a\}, \emptyset\rangle$ and $\bar{A}=\langle x, \emptyset,\{a\}\rangle$ then, $A \cap \bar{A}=\langle x, \emptyset,\{a\}\rangle \neq \widetilde{\emptyset}$ where $\widetilde{\emptyset}=$ $\langle x, \emptyset, X\rangle$. But $A \cap \bar{A} \neq \varnothing$, were A,B $\in \mathrm{T}$.

\section{Definition 2.10. [5 ]:}

Let (X,T) be an ITS and let $A=\left\langle x, A_{1}, A_{2}\right\rangle$ is said to be intuitionistic semi-open if $\mathrm{A} \subseteq \operatorname{cl}(\operatorname{int}(\mathrm{A}))$ The family of all intuitionistic fuzzy semi open sets of an ITS $(X, \tau)$ is denoted by $\operatorname{SO}(X)$.

The complement of semi open set is semi closed set .

\section{\$3- Regular in intuitionistic topological space:}

\section{Definition 3.1[4]:}

Let $\mathrm{X}$ be nonempty set and $\mathrm{T}$ is an intuitionistic topological space, then $(\mathrm{X}, \mathrm{T})$ is said to be:

1) $\mathrm{R}(\mathrm{i})$ if for each $x \in X$ and $\mathrm{F} \subseteq X, \mathrm{~F}$ is an intuitionistic closed set and $\tilde{x} \notin F$, there exist $\mathrm{U}, \mathrm{V} \in \mathrm{T}$ such that $\tilde{x} \in \mathrm{U}, \mathrm{F} \subseteq \mathrm{V}$ and $U \cap V=\widetilde{\emptyset}$. 
2) $\mathrm{R}$ (ii) if for each $x \in X$ and $\mathrm{F} \subseteq X$, $\mathrm{F}$ is an intuitionistic closed set and $\tilde{\tilde{x}} \notin F$, there exist $\mathrm{U}, \mathrm{V} \in \mathrm{T}$ such that $\tilde{\tilde{x}} \epsilon \mathrm{U}, \mathrm{F} \subseteq \mathrm{V}$ and $U \cap V=\widetilde{\emptyset}$.

3) $\mathrm{R}$ (iii) if for each $x \in X$ and $\mathrm{F} \subseteq X, \mathrm{~F}$ is an intuitionistic closed set and $\tilde{x} \notin F$, there exist $\mathrm{U}, \mathrm{V} \in \mathrm{T}$ such that $\tilde{x} \in \mathrm{U}, \mathrm{F} \subseteq \mathrm{V}$ and $U \subseteq \bar{V}$.

4) $\mathrm{R}$ (iv) if for each $x \in X$ and $\mathrm{F} \subseteq X, \mathrm{~F}$ is an intuitionistic closed set and $\tilde{\tilde{x}} \notin F$, there exist $\mathrm{U}, \mathrm{V} \in \mathrm{T}$ such that $\tilde{\tilde{x}} \epsilon \mathrm{U}, \mathrm{F} \subseteq \mathrm{V}$ and $U \subseteq \bar{V}$.

\section{Theorem 3.2[4]:}

Let $(\mathrm{X}, \mathrm{T})$ be an ITS , then the following implication are valid and the converse is not true in general :

Proof : [4]

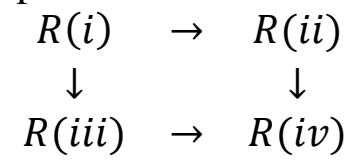

The counter example in [4]

\section{\$4- semi-Regular space in intuitionistic topological space:}

Now, we introduce new definition of semi- Regular space in intuitionistic topological space .

\section{Definition 4.1:}

Let $\mathrm{X}$ be nonempty set and $\mathrm{T}$ is an intuitionistic topological space, then $(\mathrm{X}, \mathrm{T})$ is said to be:

1) $\mathrm{SR}(\mathrm{i})$ if for each $x \in X$ and $\mathrm{F} \subseteq X, \mathrm{~F}$ is an intuitionistic semi-closed set and $\tilde{x} \notin F$, there exist $\mathrm{U}, \mathrm{V} \in$ $\mathrm{SO}(\mathrm{X})$ such that $\tilde{x} \in \mathrm{U}, \mathrm{F} \subseteq \mathrm{V}$ and $U \cap V=\widetilde{\emptyset}$.

2) $\mathrm{SR}$ (ii) if for each $x \in X$ and $\mathrm{F} \subseteq X, \mathrm{~F}$ is an intuitionistic semi-closed set and $\tilde{\tilde{x}} \notin F$, there exist $\mathrm{U}, \mathrm{V} \in$ $\mathrm{SO}(\mathrm{X})$ such that $\tilde{\tilde{x}} \epsilon \mathrm{U}, \mathrm{F} \subseteq \mathrm{V}$ and $U \cap V=\widetilde{\emptyset}$.

3) $\mathrm{SR}$ (iii) if for each $x \in X$ and $\mathrm{F} \subseteq X, \mathrm{~F}$ is an intuitionistic semi-closed set and $\tilde{x} \notin F$, there exist $\mathrm{U}, \mathrm{V} \in \mathrm{SO}(\mathrm{X})$ such that $\tilde{x} \in \mathrm{U}, \mathrm{F} \subseteq \mathrm{V}$ and $U \subseteq \bar{V}$.

4) $\mathrm{SR}$ (iv) if for each $x \in X$ and $\mathrm{F} \subseteq X, \mathrm{~F}$ is an intuitionistic semi-closed set and $\tilde{\tilde{x}} \notin F$, there exist $\mathrm{U}, \mathrm{V} \in \mathrm{SO}(\mathrm{X})$ such that $\tilde{\tilde{x}} \in \mathrm{U}, \mathrm{F} \subseteq \mathrm{V}$ and $U \subseteq \bar{V}$.

\section{Theorem 4.2:}

Let $(\mathrm{X}, \mathrm{T})$ be an ITS , then the following implication are valid and the converse is not true in general :

\section{$\underline{\text { Proof: }}$}

$\begin{array}{ccc}S R(i) & \leftrightarrow & S R(i i) \\ \downarrow & & \downarrow \\ S R(i i i) & \rightarrow & S R(i v)\end{array}$

\section{$\mathbf{S R}(\mathbf{i}) \rightarrow \mathbf{S R}($ ii)}

$\forall x \in X, \forall F \subseteq X, \widetilde{\widetilde{x}} \notin F \quad$ Sinc $(\mathrm{X}, \mathrm{T})$ is $\mathrm{SR}(\mathrm{i})$ then there exists $\mathrm{U}, \mathrm{V} \in \mathrm{SO}(\mathrm{X})$ such that $\tilde{\mathrm{x}} \in \mathrm{U}, \mathrm{F} \subseteq \mathrm{V}$, $\mathrm{U} \cap \mathrm{V}=\widetilde{\varnothing}, \quad$ We wont to prove that $\tilde{\tilde{x}} \in U, F \subseteq V$ and $U \cap V=\widetilde{\emptyset}$.

Now for each $x \in X$, for each $F \subseteq X, \tilde{\tilde{x}} \notin F, \tilde{\tilde{x}} \notin F \Rightarrow x \in F_{2},\left[\tilde{\tilde{x}} \in F, x \notin F_{2}\right]$, That's implies $\tilde{x} \in F \Rightarrow x \in F_{1}, x \notin F_{2} \Rightarrow \tilde{x} \notin F$

There exist $U, V \in \mathrm{SO}(\mathrm{X})$ such that $\tilde{x} \in \mathrm{U}$ and $\mathrm{F} \subseteq \mathrm{V}, \mathrm{U} \cap \mathrm{V}=\widetilde{\emptyset} \Rightarrow \tilde{x} \in \mathrm{U}, \mathrm{F} \subseteq \mathrm{V}, \mathrm{U} \cap \mathrm{V}=\widetilde{\varnothing}$

There for $(X, T)$ is $\operatorname{SR}(i i)$

SR(ii) $\rightarrow$ SR(i) 
Suppose $x \in X, F \subseteq X, \tilde{x} \notin F$

Sinc $(\mathrm{X}, \mathrm{T})$ is $\mathrm{SR}(\mathrm{ii})$ then there exist $\mathrm{U}, \mathrm{V} \in \mathrm{SO}(\mathrm{X})$ such that $\tilde{\tilde{\mathrm{x}}} \in \mathrm{U}$ and $\tilde{\tilde{x}} \notin F, \quad F \subseteq V, U \cap V=\widetilde{\emptyset}$.

Sinc $\tilde{\mathrm{x}} \in \mathrm{U} \Rightarrow x \in U_{2}^{c}$ thus $\tilde{\mathrm{x}} \in \mathrm{U}$ and $\tilde{x} \notin F$ and $U \cap V=\widetilde{\emptyset}$

Now $\tilde{\mathrm{x}} \in \mathrm{U} \Rightarrow x \in U_{1} \Rightarrow x \notin U_{2} \Rightarrow x \in U_{2}^{c}$. There for (X,T) is $\mathrm{SR}(\mathrm{i})$

SR(iii) $\rightarrow$ SR(iv)

Suppose $x \in X, F \subseteq X, \tilde{\tilde{x}} \notin F$

We want to prove that $U, V \in \mathrm{SO}(\mathrm{X})$ such that $\tilde{\tilde{x}} \in \mathrm{U}$ and $\mathrm{F} \subseteq \mathrm{V}, \mathrm{U} \subseteq \mathrm{V}$

Let $\tilde{\tilde{x}} \notin F \Rightarrow \tilde{x} \in F$, Sinc $(X, T)$ is $S R($ iii) then there exist $U, V \in S O(X)$ such that $\tilde{x} \in U$ and $\tilde{x} \notin F$ $, F \subseteq V, U \subseteq \bar{V}, \operatorname{sinc} \tilde{\mathrm{x}} \in \mathrm{U} \Rightarrow \tilde{\mathrm{x}} \in \mathrm{U}, \mathrm{F} \subseteq \mathrm{V}, \mathrm{U} \subseteq \overline{\mathrm{V}}$. There for (X,T) is SR(iv)

SR(i) $\rightarrow$ SR(iii)

$\forall x \in X, \forall F \subseteq X, \widetilde{x} \notin F$

Sinc $(X, T)$ is $S R(i)$ then there exists $U, V \in S O(X)$ such that $\tilde{x} \in U, F \subseteq V, U \cap V=\widetilde{\emptyset}$

We wont to prove that

$\mathrm{U} \cap \mathrm{V}=\widetilde{\varnothing}$ then $\mathrm{U} \subseteq \overline{\mathrm{V}} \quad, U \cap V=\left\langle x, U_{1} \cap V_{1}, U_{2} \cup V_{2}\right\rangle=\langle x, \emptyset, X\rangle$

Now $U_{1} \cap V_{1}=\emptyset, U_{2} \cup V_{2}=X, \quad U_{1} \subseteq \mathrm{V}_{1}^{\mathrm{c}}, \mathrm{U}_{2} \subseteq \mathrm{V}_{2}^{\mathrm{c}} \Rightarrow U \subseteq \overline{\mathrm{V}}$

There for $(\mathrm{X}, \mathrm{T})$ is $\mathrm{SR}(\mathrm{iii})$

\section{SR(ii) $\rightarrow$ SR(iv)}

$\forall x \in X, \forall F \subseteq X, \widetilde{x} \notin F, \quad \operatorname{Sinc}(\mathrm{X}, \mathrm{T})$ is $\mathrm{SR}(\mathrm{ii})$ then there exists $\mathrm{U}, \mathrm{V} \in \mathrm{SO}(\mathrm{X})$ such that $\tilde{\mathrm{x}} \in \mathrm{U}, \mathrm{F} \subseteq \mathrm{V}$, $\mathrm{U} \cap \mathrm{V}=\widetilde{\varnothing}, \quad$ We wont to prove that $\mathrm{U} \cap \mathrm{V}=\widetilde{\emptyset}$ then $\mathrm{U} \subseteq \overline{\mathrm{V}}$

$U \cap V=\left\langle x, U_{1} \cap V_{1}, U_{2} \cup V_{2}\right\rangle=\langle x, \emptyset, X\rangle$

Now $U_{1} \cap V_{1}=\varnothing, U_{2} \cup V_{2}=X \quad, \quad U_{1} \subseteq \mathrm{V}_{1}^{\mathrm{c}} \quad, \mathrm{U}_{2} \subseteq \mathrm{V}_{2}^{\mathrm{c}} \Rightarrow U \subseteq \overline{\mathrm{V}}$

There for $(\mathrm{X}, \mathrm{T})$ is $\mathrm{SR}(\mathrm{iv})$

The converse is not true in general .

Now we give examples to the reverse implications of theorem 4.2:

\section{Example 4.3:}

$\mathbf{S R}($ iv) $\rightarrow \mathbf{S R}($ iii) $\& \quad \mathbf{S R}($ iv) $\rightarrow \mathbf{S R}($ ii)

Let $X=\{a, b\}, T=\{\widetilde{\varnothing}, \tilde{X}, A, B, C\}$, where $A=\{x, \emptyset,\{a\}\}, B=\{x, \emptyset,\{b\}\} \quad, C=\{x, \emptyset, \varnothing\} \quad$, SOX $=\mathrm{T}$ $\tilde{\tilde{a}} \in X, \tilde{\tilde{a}} \notin F=\bar{A}$ such that $A, B \in S O X$, such that $\tilde{\tilde{a}} \in B \subseteq \bar{B}, F \subseteq A$ and $A \cap B=\widetilde{\emptyset}$.

$\tilde{\tilde{b}} \in X, \tilde{\tilde{b}} \notin F=\bar{B}$ such that $A, B \in S O X$, such that $\tilde{\tilde{a}} \in A \subseteq \bar{A}, F \subseteq B$ and $A \cap B=\widetilde{\varnothing}$.

Then $(\mathrm{X}, \mathrm{T})$ is $\mathrm{SR}(\mathrm{iv})$, but $(\mathrm{X}, \mathrm{T})$ is not $\mathrm{SR}(\mathrm{iii})$, because is not semi open set such that $\tilde{a} \in \mathrm{U}$.

Also (X,T) is SR(iv) but not SR(ii) because there is no semi open set $\mathrm{V}, \mathrm{U}$ such that $\tilde{\tilde{a}} \in U, F \subseteq \bar{V}$ and $U \cap V=\varnothing$.

$\mathbf{S R}($ iii) $\rightarrow \mathbf{H S R}(\mathbf{i})$

Let $X=\{a, b, c\}, T=\{\widetilde{\varnothing}, \tilde{X}, A, B, C\}$, where $A=\{x, \emptyset,\{a, b\}\}, B=\{x, \emptyset,\{c\}\} \quad, C=\{x, \emptyset, \emptyset\}$, $\mathrm{SOX}=\{\tilde{X}, \widetilde{\emptyset}, A, B, C, D, E, F, G, H, I, L, M\}$ where

$D=\{x,\{a, c\}, \varnothing\}, E=\{x,\{a, c\},\{b\}\}, F=\{x,\{b, c\}, \varnothing\}, G=\{x,\{b, c\},\{a\}\}, H=\{x, \emptyset,\{a\}\}, I=$ $\{x, \emptyset,\{b\}\}, L=\{x, \emptyset,\{a, c\}\}, M=\{x, \emptyset,\{b, c\}\}$

$(\mathrm{X}, \mathrm{T})$ is satisfy SR(iii), but is not satisfy SR(i) because

$\tilde{b} \in X, \tilde{b} \notin \bar{F}=\bar{E}$ such that $E, G \in S O X$, such that $\tilde{b} \in G, F \subseteq E$ and $G \cap E \neq \widetilde{\emptyset}$.

then $(\mathrm{X}, \mathrm{T})$ is not $\mathrm{SR}(\mathrm{i})$

Now , we give the relation of the different kinds of $\mathrm{SR}(\mathrm{k})$ where $k=\{i, i$. iii.iv $\}$ and kind of $\mathrm{R}(\mathrm{k})$ where $k=\{$ i, ii. iii. iv $\}$, which appears in definition 3.1 and definition 4.1.

\section{Theorem 4.4 :}

Let $(\mathrm{X}, \mathrm{T})$ be an ITS , then the following implication are valid and the converse is not true in general: 
$\mathrm{R}(\mathrm{i}) \rightarrow \mathrm{SR}(\mathrm{i})$

$\mathrm{R}($ ii) $\rightarrow \mathrm{SR}(\mathrm{ii})$

$\mathrm{R}($ iii $) \rightarrow \mathrm{SR}(\mathrm{iii})$

$\mathrm{R}(\mathrm{iv}) \rightarrow \mathrm{SR}(\mathrm{iv})$

Proof:

$\mathbf{R}(\mathbf{i}) \rightarrow \mathbf{S R}(\mathbf{i})$

Suppose that $x \in X$ and $F \subseteq X, \tilde{x} \in F$ since every open set is semi -open set ( ) and since (X,T) is $\mathrm{R}(\mathrm{i})$ then there exist $U, V \in T$ such that $\tilde{x} \in U, F \subseteq V$ and $U \cap V=\widetilde{\emptyset}$. Thus $\tilde{x} \in U, F \subseteq V$ and $, U, V \in$ $S O(X)$ such that $U \cap V=\widetilde{\emptyset}$. then $(\mathrm{X}, \mathrm{T})$ is $\operatorname{SR}(\mathrm{i})$

We can use similar argument to prove $\mathrm{R}($ ii) $\rightarrow \mathrm{SR}(\mathrm{ii}), \mathrm{R}($ iii) $\rightarrow \mathrm{SR}$ (iii), (iv) $\rightarrow \mathrm{SR}$ (iv)

Now we give examples to the reverse implications of theorem 4.4:

\section{Example 4.5:}

$\mathbf{S R}(\mathbf{i}) \nrightarrow \mathbf{R}(\mathbf{i})$

Let $X=\{a, b\}, T=\{\widetilde{\varnothing}, \tilde{X}, A, B, C, D, E\}$, where

$A=\{x,\{a\}, \emptyset\}, B=\{x,\{b\}, \emptyset\} \quad, C=\{x, \emptyset, \emptyset\} \quad, D=\{x, \emptyset,\{a\}\}, E=\{x, \emptyset,\{b\}\}$

$\mathrm{SOX}=\{\tilde{X}, \widetilde{\emptyset}, A, B, C, D, K, G\}$ where $, K=\{x,\{a\},\{b\}\}, G=\{x,\{b\},\{a\}\}$

$\tilde{a} \in X, \tilde{a} \notin F=\bar{K}$ such that $G, K \in S O X$, such that $\tilde{a} \in K, F \subseteq G$ and $G \cap K=\widetilde{\emptyset}$

$\tilde{b} \in X, \tilde{b} \notin F \quad$ such that $\mathrm{K}, \mathrm{G} \in \mathrm{SOX}$, such that $\tilde{\mathrm{b}} \in G, F=\bar{G} \subseteq K$ and $G \cap K=\widetilde{\emptyset}$.

Then $(X, T)$ is $S R(i)$ but not $R(i)$, if $\tilde{a} \in A, \tilde{a} \notin F$ there is no tow open set satisfy the condition $\tilde{x} \in U, F \subseteq$ $\mathrm{V}$ and $, \mathrm{U}, \mathrm{V} \in \mathrm{T}$ such that $\mathrm{U} \cap \mathrm{V}=\widetilde{\emptyset}$

\section{SR(iii) $\nrightarrow$ R(iii)}

Let $X=\{a, b, c\}, T=\{\widetilde{\varnothing}, \widetilde{X}, A, B, C\}$, where

$A=\{x, \emptyset,\{a, b\}\}, B=\{x, \emptyset,\{c\}\} \quad, C=\{x, \emptyset, \emptyset\}$,

$\mathrm{SOX}=\{\tilde{X}, \widetilde{\emptyset}, A, B, C, D, E, F, G, H, I, L, M\}$ where

$D=\{x,\{a, c\}, \emptyset\}, E=\{x,\{a, c\},\{b\}\}, F=\{x,\{b, c\}, \emptyset\}, G=\{x,\{b, c\},\{a\}\}, H=\{x, \emptyset,\{a\}\}, I=$ $\{x, \emptyset,\{b\}\}, L=\{x, \emptyset,\{a, c\}\}, M=\{x, \emptyset,\{b, c\}\}$

$(\mathrm{X}, \mathrm{T})$ is $\mathrm{SR}(\mathrm{iii})$ but its not $\mathrm{R}(\mathrm{iii})$, if $\tilde{\mathrm{a}} \in \mathrm{A}, \tilde{\mathrm{a}} \notin \mathrm{F}$ there is no tow open set satisfy the condition $\tilde{\mathrm{x}} \in \mathrm{U}, \mathrm{F} \subseteq$ $\mathrm{V}$ and $, \mathrm{U}, \mathrm{V} \in \mathrm{T}$ such that $\mathrm{U} \subseteq \overline{\mathrm{V}}$

\section{SR(iv) $\nrightarrow$ R(iv)}

Let $X=\{a, b\}, T=\{\widetilde{\varnothing}, \tilde{X}, A, B\}$, where

$A=\{x, \emptyset, \emptyset\}, B=\{x, \emptyset,\{a\}\}, \operatorname{SOX}=\{\tilde{X}, \widetilde{\emptyset}, A, B, C, D, E\}$ where

$D=\{x,\{b\}, \emptyset\}, E=\{x,\{b\},\{a\}\}, C=\{x, \emptyset,\{b\}\}$.

$\tilde{a} \in X, \tilde{a} \notin F=\bar{E}$ such that $D, C \in S O X$, such that $\tilde{a} \in C, F \subseteq D$ and $C \subseteq \bar{D}$.

$(\mathrm{X}, \mathrm{T})$ is $\mathrm{SR}(\mathrm{iv})$ but its not $\mathrm{R}(\mathrm{iv})$ if $\tilde{\mathrm{a}} \in \mathrm{A}, \tilde{\mathrm{a}} \notin \mathrm{F}$ there is no two open set satisfying the condition $\tilde{\mathrm{x}} \in \mathrm{U}, \mathrm{F} \subseteq$ $\mathrm{V}$ and $, \mathrm{U}, \mathrm{V} \in \mathrm{T}$ such that $\mathrm{U} \subseteq \overline{\mathrm{V}}$.

\section{\$5- Weak Semi Regular space in intuitionistic topological space:}

Now , we introduce new definition of weak semi regular space in intuitionistic topological space are following:-

\section{Definition 5.1:}

Let $\mathrm{X}$ be nonempty set and $\mathrm{T}$ is an intuitionistic topological space, then $(\mathrm{X}, \mathrm{T})$ is said to be:

1-WSR(i) if for each $x \in X$ and $\mathrm{F} \subseteq X, \mathrm{~F}$ is an intuitionistic semi-closed set and $\tilde{x} \notin F$, there exist $\mathrm{U}, \mathrm{V} \in$ $\mathrm{SO}(\mathrm{X})$ such that $\tilde{x} \in \mathrm{U}, \mathrm{F} \subseteq \mathrm{V}$ and $U \cap V=\widetilde{\emptyset}$. 
2-WSR(ii) if for each $x \in X$ and $\mathrm{F} \subseteq X, \mathrm{~F}$ is an intuitionistic semi-closed set and $\tilde{\tilde{x}} \notin F$, there exist $\mathrm{U}, \mathrm{V} \in$ $\mathrm{SO}(\mathrm{X})$ such that $\tilde{\tilde{x}} \epsilon \mathrm{U}, \mathrm{F} \subseteq \mathrm{V}$ and $U \cap V=\widetilde{\emptyset}$.

Now, we give the relation of the different kind of $\operatorname{WSR}(\mathrm{k})$ where $\mathrm{k} \in\{\mathrm{i}$, ii $\}$ and kinds of WR(k) where $\mathrm{k} \in\{\mathrm{i}, \mathrm{ii}\}$, which appears in definition 4.1 and definition 3.1 in [4]

\section{Theorem 5.2[4]:}

Let $(\mathrm{X}, \mathrm{T})$ be an ITS , then the following implication are valid and the converse is not true in general :

\section{Proof:}

$\begin{array}{ccc}S W R(i) & \leftrightarrow & S W R(i i) \\ \downarrow & & \downarrow \\ W R(i) & \rightarrow & W R(i i)\end{array}$

\section{SWR(i) $\rightarrow$ SWR(ii)}

Let $(\mathrm{x}, \mathrm{t})$ is satisfies WSR(i) if for each $x \in X, F \subseteq X, F$ is ISCS and $\tilde{x} \notin F$ there exists $U, V \in S O(X)$ such that $\tilde{x} \in U, F \subseteq V$ and $U \cap V=\emptyset$. also since $\tilde{x} \in U \Rightarrow \tilde{\tilde{x}} \in U$ from the theorem 4.2 .

SW R(ii) $\rightarrow$ SWR(i)

Let $(\mathrm{x}, \mathrm{t})$ is satisfies WSR(ii) if for each $x \in X, F \subseteq X, F$ is ISCS and $\tilde{\tilde{x}} \notin F$ there exists $U, V \in S O(X)$ such that $\tilde{\tilde{x}} \in U, F \subseteq V$ and $U \cap V=\emptyset$.

Now $\tilde{\tilde{x}} \in U \Rightarrow x \in U_{2}^{c} \Rightarrow x \notin U_{2} \Rightarrow x \in U_{1}$ Thus $\tilde{x} \in U, F \subseteq V$ and $U \cap V=\varnothing$ there for (X,T) is WSR(i).

$\mathbf{W} \mathbf{R}(\mathbf{i}) \rightarrow \mathbf{S W R}(\mathbf{i})$

Let $(\mathrm{x}, \mathrm{t})$ is satisfies WR(i) if for each $x \in X, F \subseteq X, F$ is ISCS and $\tilde{\tilde{x}} \notin F$ there exists $U, V \in T$ such that

$\tilde{\tilde{x}} \in U, F \subseteq V$ and $U \cap V=\emptyset$. since every $\operatorname{WR}(\mathrm{k})$ where $\mathrm{k} \in\{\mathrm{i}, \mathrm{ii}\}$ is $\operatorname{WSR}(\mathrm{k})$ where $\mathrm{k} \in\{\mathrm{i}, \mathrm{ii}\}$. Thus $(\mathrm{X}, \mathrm{T})$ is satisfies WSR(i).

In asimilar way we can prove $\mathbf{W} \mathbf{R}($ ii) $\rightarrow \mathbf{S W R}(\mathbf{i i )}$.

$\mathbf{W}$ R(i) $\rightarrow$ W R(ii)

This given in [4]

The reverse implication in theorem 5.2 are not true in general ,the following counter example shows the cases

\section{Example 5.3:}

Recall example 4.5 we see that

$\operatorname{SWR}(\mathbf{i}) \nrightarrow \mathbf{W R}(\mathbf{i})$

$(\mathrm{X}, \mathrm{T})$ is WSR(i), but not WR(i) .

SWR(ii) $\nrightarrow$ WR(ii)

$(\mathrm{X}, \mathrm{T})$ is WSR(ii), but not WR(ii) .

\section{Example 5.4:}

WR(ii) $\rightarrow$ WR(i)

This given in [4]

\section{References}

[1] S. Bayhan and D. Coker, On separation axioms in intuitionistic topological spaces, Int.

J. Math. Math. Sci. 27 (2001), no. 10, 621-630.

[2] Coker, D. An introduction to intuitionistic fuzzy topological space. Fuzzy sets and systems, 88:81:89, 1997.

[3] Coker, D. An introduction to intuitionistic topological spaces, BUSEFAL 81, 51 \{56, 2000.

[4] Y.Yaseen and A.Mahammad, On regular and weak regular space in intuitionistic topological spaces, J. Of university of anbar for pure science ,2011. 